\title{
Search for CVs in wide-field narrow-band photometric survey
}

\section{Javier Abril*}

Centro de Estudios de Física del Cosmos de Aragón

E-mail: jabrilecefca.es

\section{Alessandro Ederoclite}

Centro de Estudios de Física del Cosmos de Aragón

\section{Linda Schmidtobreick}

European Southern Observatory

Cataclysmic Variables (CVs) are binary systems made of a white dwarf which is accreting mass from a less evolved companion. Depending on the physical properties of the system, the observational characteristics of CVs can be very diverse. Nevertheless, as we learned from projects like the Sloan Digital Sky Survey, CVs occupy the same locus of quasars in color-color diagrams, hence their discovery can be quite challenging. In this paper, we expose how the filter set of the J-PLUS project can help to efficiently separate CVs from other objects (mostly quasars) and even identify their type. Through simulations and real data, we explain how accurate the method is and identify the following steps to finally get the first complete unbiased magnitude-limited sample of Cataclysmic Variables to date, a fundamental data set to be able to study the evolution of this type of objects.

The Golden Age of Cataclysmic Variables and Related Objects IV 11-16 September, 2017

Palermo, Italy

\footnotetext{
* Speaker.
} 


\section{Introduction}

Cataclysmic variables (CVs) are interacting binary stars made of a white dwarf and a main sequence star which is transferring mass via Roche lobe overflow. Since the structure of both components is relatively simple, $\mathrm{CVs}$ are one of the best sources to test our understanding of many astrophysical phenomena involving evolution of compact, interacting binaries. At present, there are a number of standing discrepancies between current $\mathrm{CV}$ population models and observations. It is fundamental to solve these disagreements in order to validate the application of these models to many other present and complex topics such as black hole binaries, short gamma-ray bursts, X-ray transients, milli-second pulsars and Supernovae Ia. For a complete review, see Warner (1995).

The evolution of such binaries is driven by angular momentum loss, which controls the change of orbital period as well as the mass transfer rate. The orbital period distribution is the main tool to study the evolution of $\mathrm{CVs}$, as the orbital period is the easiest parameter to determine for these binaries. During their evolution, CVs move from long orbital periods and high mass transfer rates to short orbital periods and low mass transfer rates. The evolution proceeds this way until the system reaches the period minimum at $\sim 78 \mathrm{~min}$ in which the donor mass has become so low that core H-burning ceases, and the star turns into a brown dwarf (BD). Consequently, the orbital separation and period now increases as the mass transfer continues. These systems are called period bouncers. Theory predicts that $70 \%$ of the CVs (Kolb 1993) should belong to the period bouncers, faint systems with short orbital periods. Instead, observations show an overabundance of bright, high mass transfer systems at periods between about 3 and 4 hours (Schmidtobreick 2017) and no period bouncer has been unambiguously identified yet.

Moreover, observations show an abrupt drop in the number of systems with periods between 2 and $3 \mathrm{~h}$, referred to as the period gap. Below this range (Porb $<2 \mathrm{~h}$ ) systems have low mass-transfer rates governed by gravitational radiation while the higher mass-transfer rates above the gap (Porb $>3 \mathrm{~h}$ ) are a consequence of the stronger magnetic braking. The standard explanation suggests that magnetic braking switches off when a CV has evolved down to $3 \mathrm{~h}$, the secondary contracts to its thermal equilibrium and detaches from its Roche lobe. The continuing angular momentum loss by gravitational radiation shrinks the orbit until at a period of about $2 \mathrm{~h}$, the Roche lobe makes contact with the stellar surface and mass transfer is re-established albeit at a lower level. Several explanations have been proposed to explain why the magnetic braking switches off. Possibly, at the mass of a Roche-lobe-filling secondary in a $\sim 3 \mathrm{~h}$ binary, the red dwarf becomes fully convective, and this might quench the magnetic dynamo. Arguing against this is the fact that signs of magnetic activity are still observed in lower-mass single stars.

In both cases the discussion is on-going and only a well defined sample of CVs can help to disentangle these discrepancies between theory and observations.

The only complete sample of CVs that we are aware of (Pretorius, Knigge 2012; Pretorius et al. 2013) is too small to answer these questions. The aim of this project is to enlarge the sample of well characterized CVs in order to carry out a stringent test on the present models of CV evolution. 


\section{Essence of the project}

\subsection{Javalambre-Photometric Local Universe Survey, J-PLUS}

Historically, CVs have been discovered through their variability properties and, most recently, as by-product of quasar searches (see Gaensicke 2005). In fact, SDSS alone discovered more than 400 new CVs (Szkody et al., 2011). In general terms, a CV spectrum can be blue and/or red and it often shows $H_{\alpha}$ emission arising from the accretion disk. Since $H_{\alpha}$ line emission in quasars is red-shifted according to the expansion of the Universe, the relation between the line emission $H_{\alpha}$ and the filter rSDSS is a powerful tool for an early discrimination between CVs and quasars.

Nonetheless, since quasar spectral energy distributions are red-shifted, they can display a variety of observational properties making their distinction more convoluted and often broad band filters do not provide the necessary information. In contrast, J-PLUS (Cenarro et al., in prep. ) has a set of 12 broad, intermediate and narrow band optical filters, specifically designed to cover key spectral features for stellar classification. A relevant example is the case of J0660, a narrow-band filter centered at the rest-frame wavelength of the $H_{\alpha}$ line. J-PLUS is an unprecedented photometric sky survey of $8000 \mathrm{deg} 2$ reaching AB-magnitude 21.5. Given its unique set of filters, J-PLUS is in a remarkably good position to identify CVs which have gone unnoticed so far.

\subsection{Background in the search of CVs}

The spectral energy distribution (SED) of CVs is the result of the superposition of the spectra of a WD, a main sequence star and, depending on the accretion rate and the magnetic field of the WD, an accretion disc. It is evident that the SED of a CV will be very different from the SED of isolated non-accreting stars. Given their nature, CVs have been discovered in very diverse ways. As mentioned before and as their name suggests, the search for CVs greatly benefited from serendipity findings based on their variability properties. For example, the Catalina Real-time Transients Survey (CRTS) has reported one thousand CVs (Breedt et al. 2014). More recently, with the arrival of large field surveys like the $2 \mathrm{dF}$ or the SDSS, CVs were found as by-product of quasar searches since they tend to cover the same locus in color-color diagrams based on broadband, optical filters. In the last years, some attempts have been made to identify CVs based on $H_{\alpha}$ excess and WISE colors (Scaringi et al. 2013) or SDSS colors and proper motions (Gentile-Fusillo et al. 2015).

\section{Deriving Color-Cuts}

Depending on the mass transfer rate, the magnetic activity of the white dwarf and the inclination of the system, CVs can display a variety of observational properties. Roughly speaking, a $\mathrm{CV}$ spectrum can be blue and/or red in function of the mass transfer rate and it uses to show $H_{\alpha}$ emission due to the accretion disk. High mass transfer CVs spectra tend to be dominated by the disk contribution, hence they often show strong $H_{\alpha}$ emission lines along with flatter continuum where the secondary is rarely visible.

Quasars at low redshifts are mostly blue while they become redder as their redshifts increases. According to the redshift of the quasar, some line may be located at the same wavelength of $H_{\alpha}$ at rest frame (e.g. $O I I I$ and $H_{\beta}$ at $z \sim 0.35, C I I I$ at $z \sim 2.4$ or $C I V$ at $z \sim 3.25$, see fig. 1). 


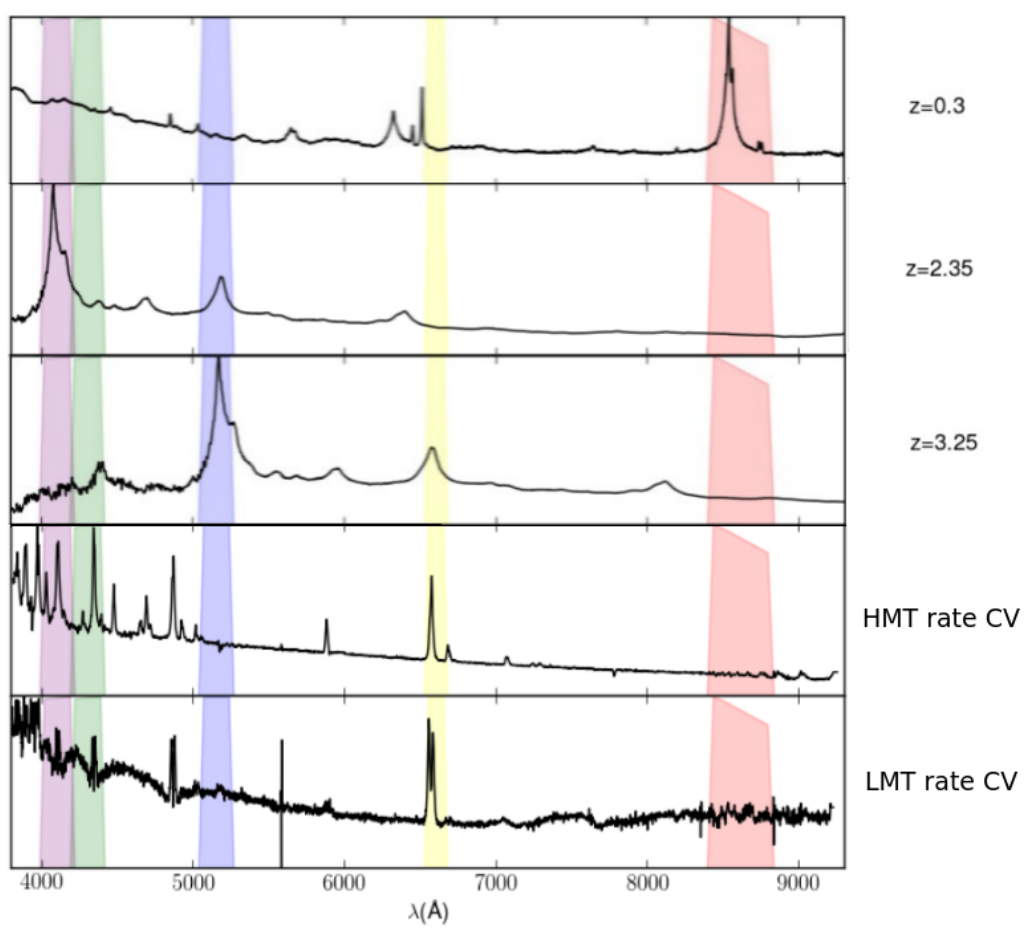

Figure 1: Comparative spectra of three Quasars at different redshifts, the top three SEDs, and 2 CVs at the bottom, one of them a high mass transfer $\mathrm{CV}$ and a low mass transfer $\mathrm{CV}$ the other. We can see there some tricky redshifts in which an emission line gets into the J0660 filter so their discrimination needs of other color-color combination such as colors involving iSDSS, zSDSS or the JPLUS narrow filter J0861.

We have investigated the possibility to improve the method proposed by Scaringi et al (2013), involving $H_{\alpha}$ excess and WISE colors, which lead us to the development of a method based on three color-color cuts which aims at identifying CVs. As shown in the first panel of fig. 2, most of high mass transfer $\mathrm{CVs}$ fall in the shaded region, with the contamination of a small portion of quasars at specific redshift mentioned above. Since the higher the redshift the redder the spectrum, the contribution in the UJAVA filter is small compared to redder regions of the spectrum and therefore, quasars at $z \sim 2.4$ and $z \sim 3.25$ are distinguishable using $u J A V A-i S D S S$ color (there are no synthetic quasars at these values but there are some examples in fig. 4). On the other hand, lower redshifts ranges as $z \sim 0.35$ introduce $H_{\alpha}$ line emission in the zSDSS filter, and so, comparison with the adjacent iSDSS allows their discrimination (last panel of the same figure).

On the other hand, low Mass Transfer (LMT) CVs are located in the unshaded part inside the cut in the first panel of fig. 2. Since their spectra is not dominated by the disk, the secondary is detectable in the infrared through the WISE filters WISE1 - WISE2, as shown in the central panel of the same figure.

To test the validity of this methodology, first we applied it to 9255 quasars, selected as being classified as point-like sources, and $121 \mathrm{CVs}$ whose spectroscopy was available from SDSS. After convolving with the J-PLUS filter system, we could recover 109 of the CVs, therefore obtaining a 
completeness of $90 \%$, and only 4 quasar contaminants.
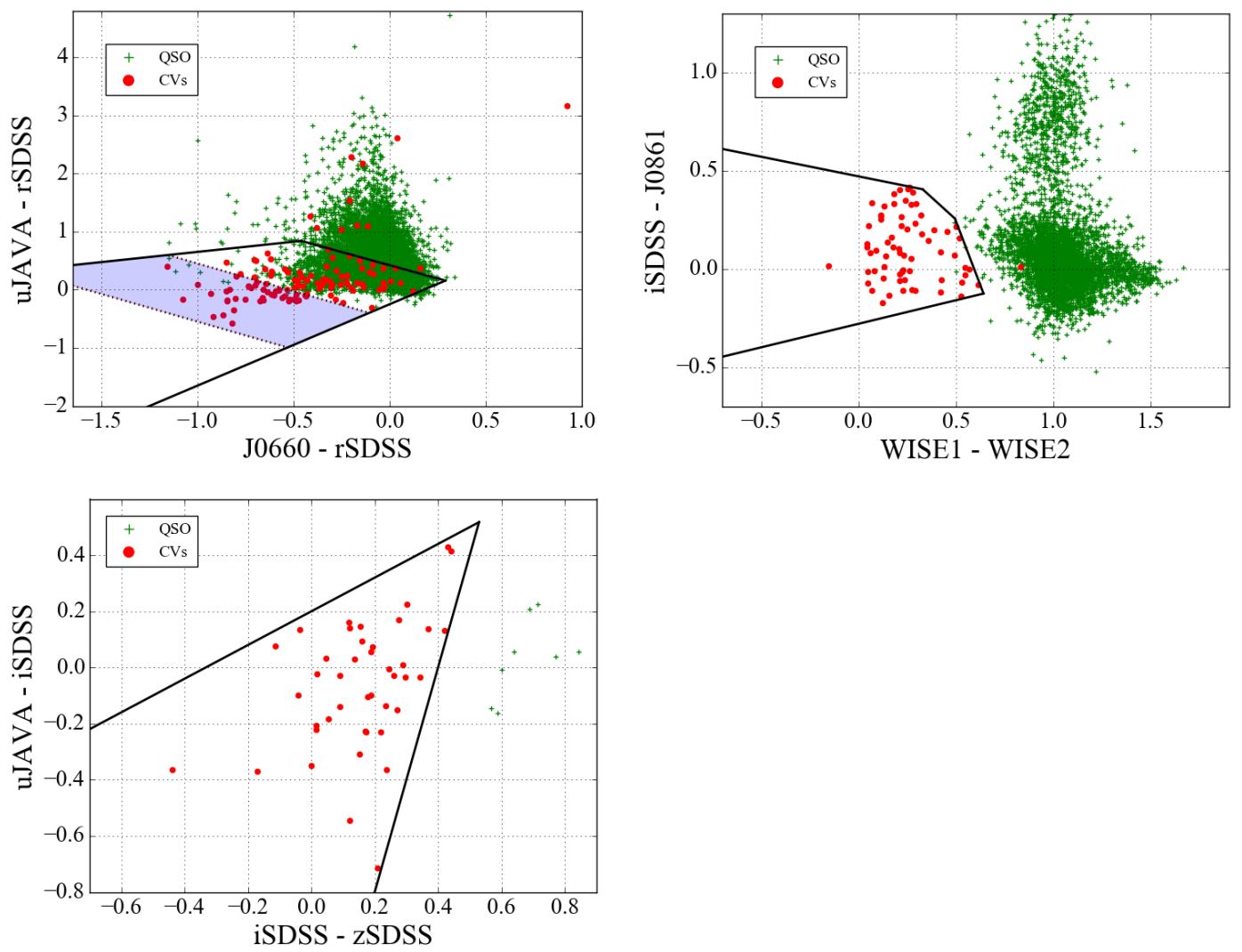

Figure 2: Three color-cuts devised to disentangle CVs from quasars, the red dots are the CVs and the green crosses are the Quasars. Top-left panel shows the color-color diagram J0660-rSDSS vs uJAVA-rSDSS. Most of the CVs lie within the black borders due to the $H_{\alpha}$ emission combined with low $u J A V A-r S D S S$, but there are also two well-defined regions inside. The unshaded part contains low mass transfer CVs and a big amount of quasars of different redshifts. Top right panel collects all these objects from the unshaded part on top left panel and compares WISE1 - WISE2 to iSDSS - J0861, all with the aim of detecting the secondary of the CVs and nonexistent on quasars and thus recover the CVs. High mass transfer CVs and some quasars at very specific redshifts are located on the shaded part of top right panel. Bottom left panel takes these sources and shows how low redshift quasars as $z \sim 0.35$ display higher $i S D S S-z S D S S$ color than CVs since quasars have $H_{\alpha}$ redshifted to the $z S D S S$ band. The $u J A V A-J 0861$ is faced to make CVs distinguishable from higher, also complicated redshifts as $z \sim 2.4$ and $z \sim 3.25$. Since these kind of quasars are redder due their high redshifts, the color $u J A V A$ - iSDSS tends to be lower on CVs (even though there are no such examples on these diagrams).

Later, we checked the goodness of our method with real J-PLUS data, concretely with a first internal release of 150 square degrees and more than 18000 objects cataloged as punctual sources, and with restrictions in magnitude and $\mathrm{S} / \mathrm{N}$, fig. 4 . This time we recovered the $100 \%$ of the known $\mathrm{CVs}$ in it (7/7) and had 20 candidates/contaminants, 4 cataloged as quasars, 3 as galaxies and 13 as stars.

It is noteworthy that several of these contaminant stars are binary systems composed by a white dwarf and a main sequence star (WD+MS, period gap CV candidates) or a white dwarf and a brown dwarf (WD+BD, period bouncer $\mathrm{CV}$ candidates) and it seems we have strong candidates 


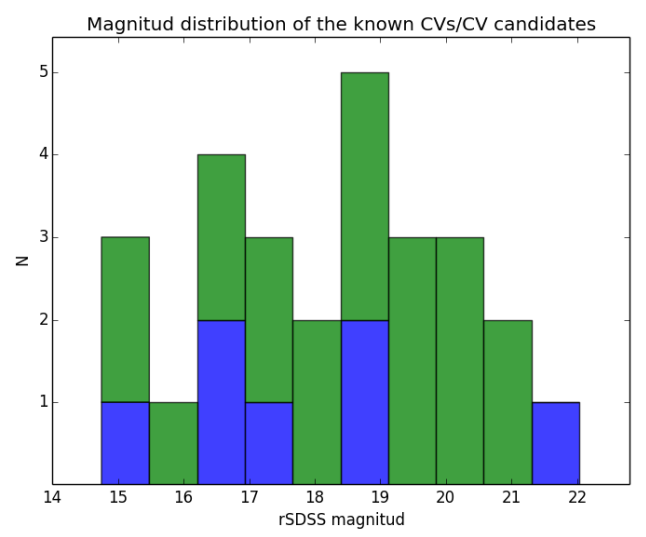

Figure 3: In total there are $20 \mathrm{CV}$ candidates in green and 7 known CVs in blue, with magnitudes in the range between 15 and 22 .

to help solving directly the CV evolution discrepancies mentioned above. The figure 5 shows two examples of period gap candidates, our methodology classifies them as CVs and the lack of emission lines, meaning they don't have accretion, makes them strong period gap candidates.

\section{Summary and Conclusions}

We have presented a new methodology, based on the J-PLUS filter set and WISE, to identify $\mathrm{CVs}$ in wide field surveys. The methodology is based on cuts in color-color diagrams. As the result of applying this methodology to the first J-PLUS Internal Data Release (IDR) of 150 square degrees, we have obtained a completeness of $100 \%$, recovering the 7 known CVs and only $22 \mathrm{CV}$ candidates/contaminants out of 18000 objects. The methodology is biased to non-magnetic CVs and our main contaminant is quasars with redshift around 2.2, more efforts must be done in order to improve the selection criteria in this direction. However, among our contaminants we also have WD+MS and WD+BD binary stars, period bouncers and period gap candidates, which can lead to resolve theory evolution discrepancies of CVs. Since we expect to have about 1000 quasars for each CV in the J-PLUS survey, both completeness and purity are very promising to be able to provide an unbiased magnitude-limited sample of CVs for evolutionary studies. The next step is their spectroscopic confirmation in order to fully validate the methodology. We also have embarked on a new approach based on machine learning algorithms which we expect to improve our selection and, most interestingly, the characterization of their types and subtypes.

\section{Acknowledgments}

Funding for the J-PLUS Project has been provided by the Governments of Spain and Aragón through the Fondo de Inversiones de Teruel, the Spanish Ministry of Economy and Competitiveness (MINECO; under grants AYA2015-66211-C2-1-P, AYA2015-66211-C2-2, AYA2012-30789 and ICTS-2009-14), and European FEDER funding (FCDD10-4E-867, FCDD13-4E-2685). The Brazilian agencies FAPESP and the National Observatory of Brazil have also contributed to this project. 

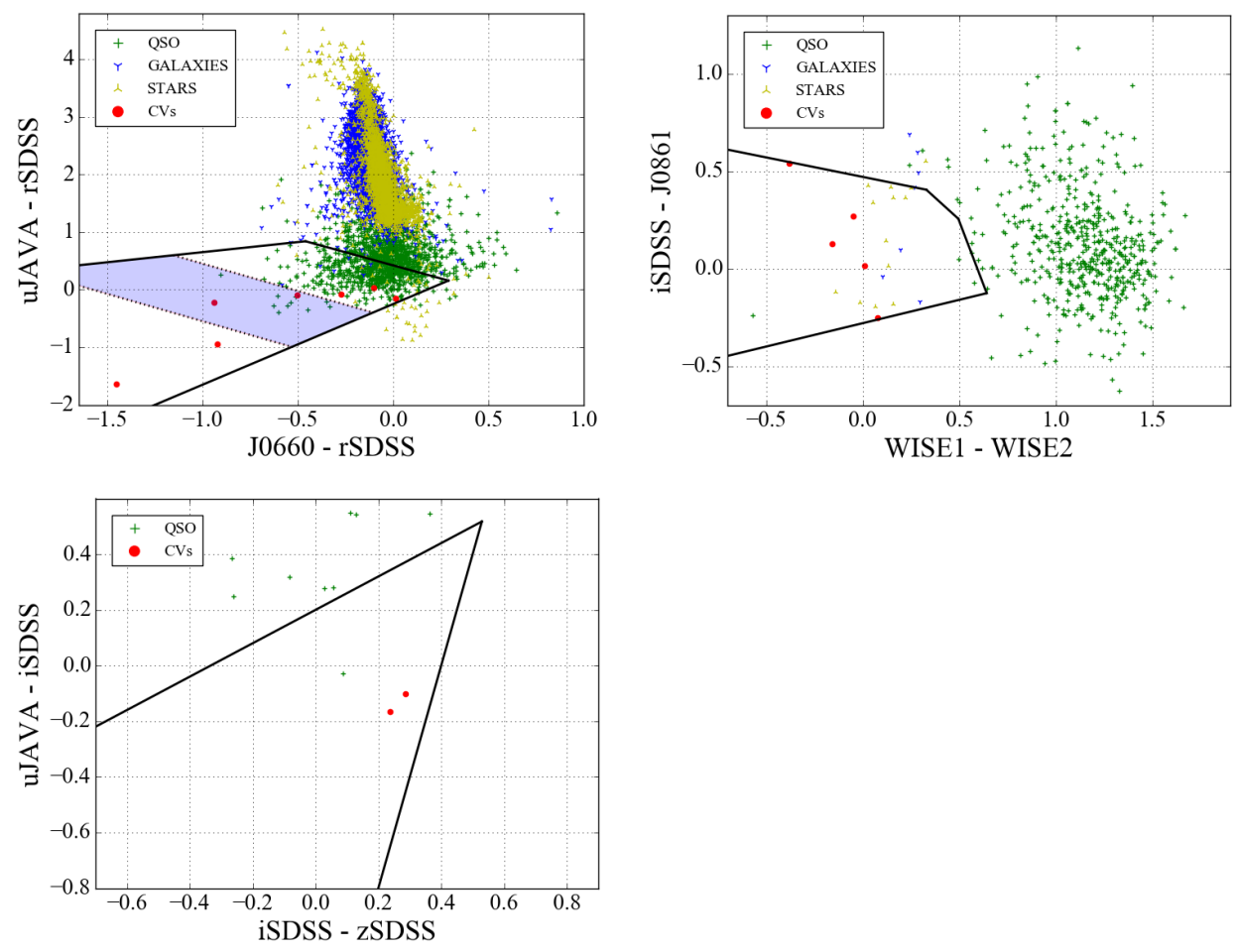

Figure 4: The three color cuts applied to the first 150 square degrees of J-PLUS. The different symbols refer to the classification given by SDSS for objects with J-PLUS photometry. The objects falling in the shaded area in the left panel are evaluated again in the bottom one. The objects out of the shaded area but within the cuts are evaluated in the right panel.
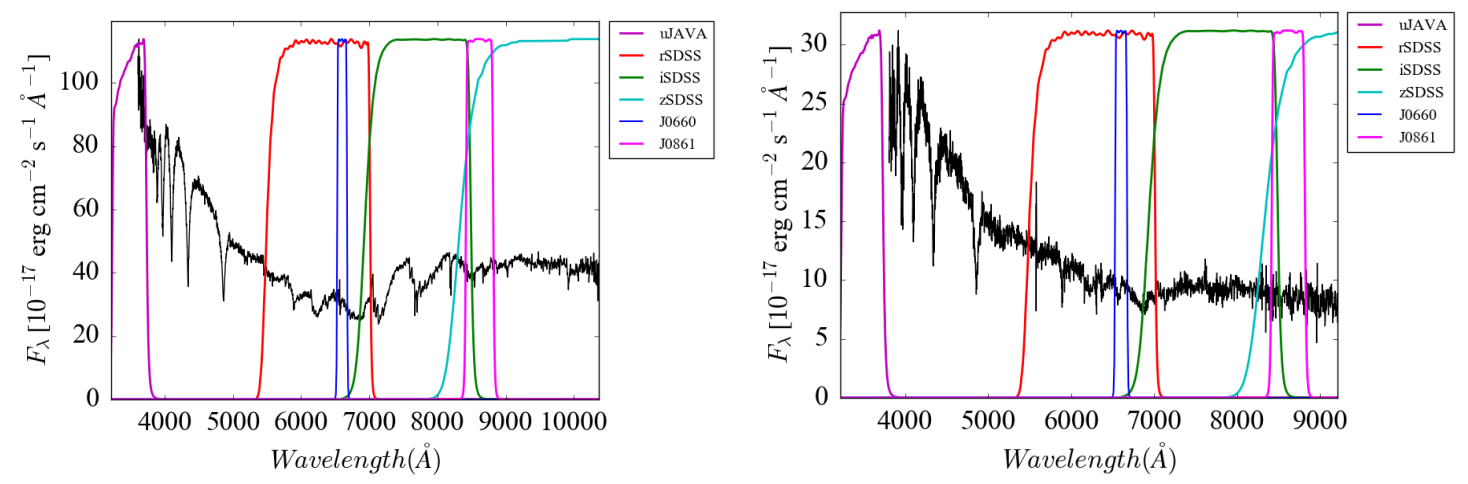

Figure 5: The figure shows two examples of period gap candidates, our methodology classifies them as CVs and the lack of emission lines, meaning they don't have accretion, makes them strong period gap candidates.

\section{References}

[1] Breedt et al. 2014, MNRAS, 443, 3174

[2] Cenarro et al. in prep.

[3] Gaensicke 2005, ASPC, 330, 3

[4] Gentile-Fusillo et al. 2015, MNRAS, 448, 2260 
[5] Kolb 1993A\&A, 271, 149

[6] Pretorius, Knigge 2012, MNRAS 419,1442

[7] Pretorius et al. 2013, MNRAS 432, 570

[8] Scaringi et al. 2013, MNRAS, 428, 2207

[9] Schmidtobreick 2017

[10] Szkody et al., 2011, AJ, 142,181

[11] Warner Cataclysmic Variable Stars 1995, Cambridge University Press 